\title{
Rationale for vacuum-pulse pump devices applied on cattle farms
}

\author{
Insaf Nafikov*, Rail Khusainov, Ruslan Lukmanov, Ilgiz Galiev and Ilnur Gayfullin \\ Kazan State Agrarian University, Kazan, Russia
}

\begin{abstract}
One of the main elements to foster technological processes in animal husbandry are vacuumpulse pump devices designed to support dosing, mixing, transporting, lines for forage preparation and feeding, milking cows, milk processing, as well as a large number of technological processes in agriculture. The paper discusses a vacuum-pulse pumping device widely used in all industries and agriculture, a feature of which is to improve technical characteristics of the pump avoiding direct mechanical energy consumption and boasting a fairly simple design. In vacuum-pulse pumping devices, transient events are caused deliberately to increase ejection coefficient, productivity, etc. Oscillating flows of materials being transported are very diverse, due to an increased number of similarity criteria that determine flow patterns. Whereas superficial velocity and the Reynolds number are commonly used for a steady flow, for an oscillating flow, the relative frequency and the relative amplitude of oscillations are added. The objects of experimental research were ejectors with oscillating flows. The wider objective of the experiments was to determine the most effective performance indicators of the ejectors, including the degree of pressure increase, the ejection coefficient and the geometric parameter. Resulting from the experiments, a direct relationship was established between changes in the performance of a pulse-vacuum pumping device and valve material and magnitude of its oscillations. A pulse ejector is recommended to have metal-seated ball valves with a pulsation frequency of $90-100 \mathrm{~min}^{-1}$. Once applied, the proposed pulse ejector will eventually increase the transportation productivity by $14.5 \%$.
\end{abstract}

\section{Introduction}

An integral part of the farming industry is high-quality agricultural production, which is related to mechanization of technological processes [1-4]. Today, effective engineering solutions are being sought to improve these processes [5-8].

One of the key elements to foster technological processes in animal husbandry are vacuum-pumping devices designed to support dosing, mixing, transporting, drying, lines for forage preparation and feeding, milking cows, milk processing, as well as a large number of technological processes in agriculture $[9,10]$. One of the disadvantages of the existing vacuum-pumping devices is that the output parameters are limited by the technical characteristics of the pump [11-13].

One of the ways to increase the efficiency of vacuum pumping devices for mixing, dosing gaseous media and bulk materials is reported to be an intermittent (oscillating) flow [14].

The use of eductor-jet pumps (ejectors) can, along with a decreased residual pressure, significantly reduce energy costs, irrespective of the dimensions.

The reliance on transient flows promotes more efficient energy exchange between motive and passive media.
The effectiveness of the method for influencing the medium, the parameters of which depend on time, can be attributed to the possibility of transferring motion from the motive fluid to the passive medium through pressure waves, as well as by organizing the process with serial and parallel connection of additional mass [14].

The efficiency of ejectors with motive transient flows in comparison with the same ejectors with steady flows showed an increase in the ejection coefficient by a factor of $1.5-2$ [14].

\section{Material and methods}

An oscillating motive fluid flowing to a mixing chamber can be achieved by occasionally opening and closing a flow section of a jet nozzle, which is done with the help of regulating elements performing reciprocating and translational motion (valve-type ejectors), as well as rotational motion (focal-slot and drum type devices). The most economically feasible in agricultural conditions are valve-type ejectors (Fig. 1) [15].

\footnotetext{
* Corresponding author: insaf-82@mail.ru
} 


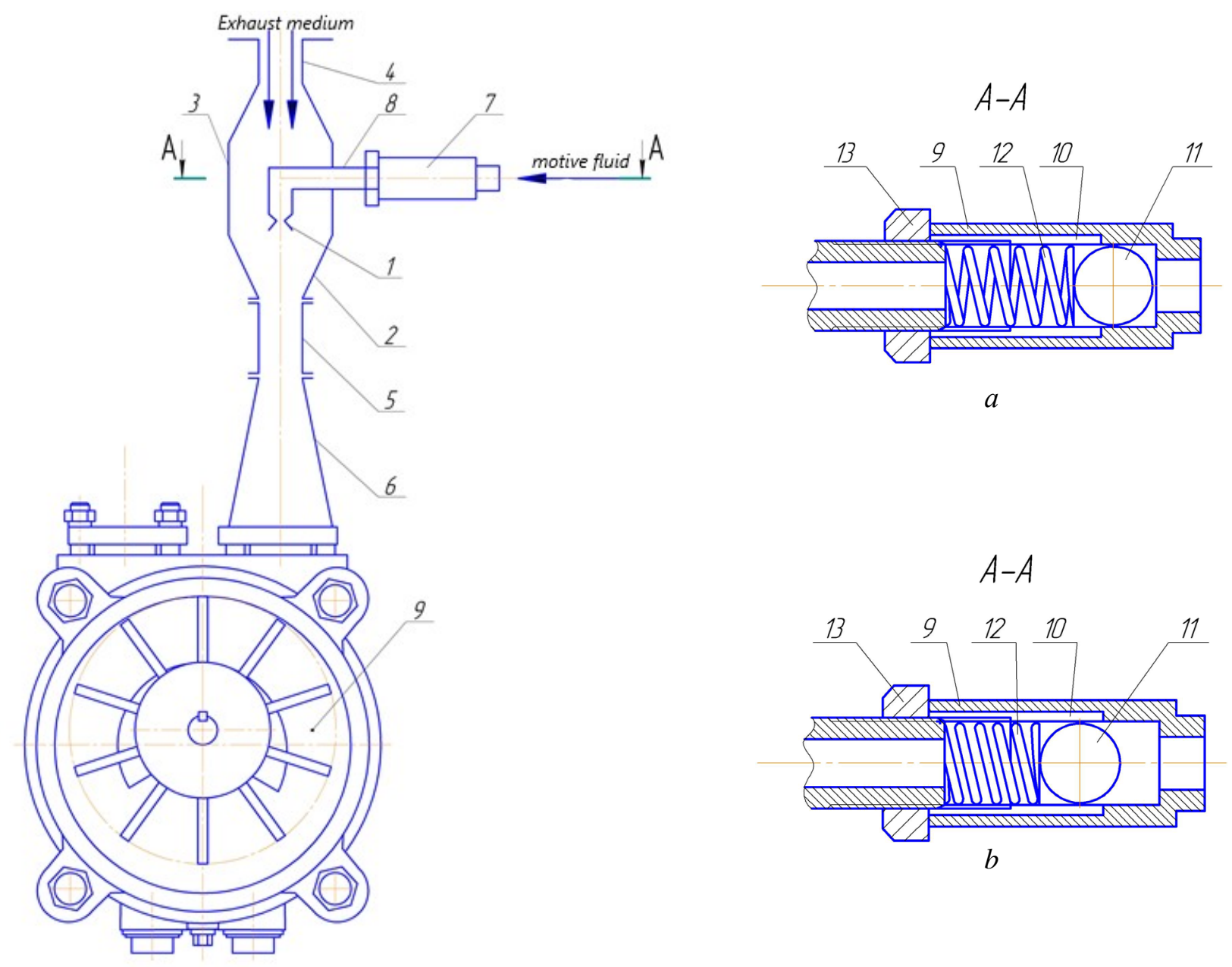

Fig. 1. Schematic for a pulse ejector as part of water-packed vacuum pump: a - make-and-break diagram at the moment of closing a motive medium inlet; $b$ - make-and-break diagram at the moment of opening a motive medium inlet; 1 - high-pressure nozzle; 2 exhaust medium; 3 - receiving chamber; 4 - pipe for exhaust medium; 5 - mixing chamber; 6 - diffuser; 7 - make-and-break mechanism; 8 - inlet pipe; 9 - water-packed vacuum pump (WPVP)

Figure 1 shows a diagram of a pulse ejector as part of the WPVP. The pulse ejector includes a high-pressure nozzle 1 for supplying a motive fluid, made either of a narrowing or expanding form (depending on the aggregation of the motive fluid). In a supply line, at the central motive-fluid nozzle, a make-and-break mechanism is installed, which consists of a cylindrical sleeve, where channels are provided for supplying a high-pressure medium (Figure $1 a, b$ ). A ball and a spring are provided inside the sleeve to ensure highpressure flow intermittent. The inlet pipe is opened by a high-pressure flow acting on the ball. The inlet is closed with the same ball due to the force action from a cylindrical spring, the elasticity of which is regulated by the sleeve being screwed onto the inlet pipe of the eductor-jet pump.

To provide a rationale for certain design parameters of the pulsator, it is necessary to establish a relationship between pulsation frequencies on the effectiveness of technological operations.

Generally speaking, to consider motion, onedimensional unsteady equations of gas dynamics include the equations of flow rate-continuity (1), conservation of momentum (2) and energy (3) [16].

$$
\begin{gathered}
\frac{\partial}{\partial \tau}(\rho S)+\frac{\partial}{\partial x}(\rho V S)=0 \\
\frac{\partial}{\partial \tau}(\rho V S)+\frac{\partial}{\partial x}\left[S\left(\rho V^{2}+p\right)\right]=p \frac{\partial S}{\partial x}-\frac{\partial}{\partial x} \Delta I, \\
\frac{\partial}{\partial \tau}\left[\rho S\left(e+\frac{V^{2}}{2}\right)\right]+\frac{\partial}{\partial x}\left[\rho V S\left(e+\frac{p}{\rho}+\frac{V^{2}}{2}\right)\right]=0,
\end{gathered}
$$

where $\tau$ - time,

$x$ - longitudinal coordinate,

$\rho$ - gas density,

$p$ - pressure,

$V$ - flow rate,

$S$ - channel cross-sectional area,

$e$ - base of natural logarithm, $e=2,73$,

$\Delta I$ - frictional gas energy losses and local losses.

Time variation of static pressure $p(\tau)$, flow or pressure regulation is usually done by changing the flow area $F(\tau)$. It is envisaged that the pressure and the flow area change according to a harmonic law (4). With the harmonic law, the amplitude, frequency and phase angle are set, and the change in pressure at the boundary is determined as 


$$
p=p_{\text {avg }}+p_{\text {max }} \sin \left(2 \pi f \tau+\varphi_{0}\right),
$$

where $p_{\text {avg }}$ is the average pressure in the pulsator, $\mathrm{Pa}$;

$p_{\max }$ is the amplitude value of the pressure in the pulsator, $\mathrm{Pa}$;

$f$ is pulsation frequency, $\mathrm{s}^{-1}$;

$\varphi_{0}$ is the initial phase.

To increase the ejection ratio and maintain a high compression ratio in the ejector, the mixing chamber is made in the form of a cylindrical throat.

Based on this, the average pressure in the mixing chamber and the pressure amplitude in the pulsator were found to determine the pressure in the exhaust medium. Then the formula for the pressure of the exhaust medium will look like this.

$$
p_{\text {avg }}=\frac{p^{*}}{\frac{\sqrt{(u+1)(1+u \theta+\vartheta)}}{1+1 / \alpha} \frac{q\left(\lambda_{1}\right)}{q\left(\lambda_{3}\right)}},
$$

where $p^{*}$ is the total gas pressure;

$u$ is the ejection coefficient, $m_{2} / m_{1}$;

$m_{2}$ is the mass of gas at the outlet of the ejector;

$m_{l}$ is the mass of gas at the inlet;

$\theta$ is the ratio of temperatures in the ejector;

$\alpha$ is the geometric factor $\alpha=\mathrm{F}_{1} / \mathrm{F}_{2}$;

$F_{1}$ is the outlet area of the motive-fluid nozzle;

$\mathrm{F}_{2}$ is the outlet area of the passive nozzle;

$q(\lambda)$ is the gas dynamic function of the flow rate.

Based on formula 5, equation 4 will take the form:

$$
p_{\Sigma}=\frac{p^{*}}{\frac{\sqrt{(u+1)(1+u \theta+\vartheta)}}{1+1 / \alpha} \frac{q\left(\lambda_{1}\right)}{q\left(\lambda_{3}\right)}}+p_{\max } \sin \left(2 \pi f \tau+\varphi_{0}\right) \cdot(6)
$$

This formula describes all processes as long as vacuum ejectors are used with a pulsator.

Preliminary calculations show that there is a boundary between velocities and pressures, where the effect of pulsation is not significant. However, in a wide range of variation of these parameters, especially in the zone of supersonic flows, the pulsation effect is significant.

The paper is concerned with ejectors working in onedimensional theory of flow motion, since the complexity of mathematical solutions for two-dimensional flows makes these problems unsolvable. In addition, the results of the calculations performed according to onedimensional theory are in good agreement with the experimental data.

The objects of experimental research are pulse ejectors. The amount of vacuum was calculated using the optimization methods developed at the Kazan State
Agrarian University. The objective of research was to determine and prove the key parameters of a vacuum ejector, as well as to experimentally confirm the reliability of research results. The wider objective of experiments was to determine the most effective performance indicators of ejectors including the degree of pressure increase, ejection coefficient and geometric parameter. Experimental studies were carried out according to the developed algorithm using a VVN-0.25 water-packed vacuum pump. Each parameter was measured 3 times, and their average value was taken.

\section{Results and Discussion}

As a result of experimental research, a pulse ejector, in addition to the parameters mentioned above, is greatly subject to a valve (shape and material) selected, providing an intermittent supply of a motive flow.

As a result of joint mathematical processing of the experimental results, the dependences of productivity on the value of vacuum were obtained for various ball materials (metal, rubber and textolite):

$$
\begin{aligned}
& \mathrm{Q}_{\mathrm{M}}=6.8541+0.3659 \cdot \mathrm{P}-0.0038 \cdot \mathrm{P}^{2}, \\
& \mathrm{Q}_{\mathrm{r}}=4.6446+0.3874 \cdot \mathrm{P}-0.0039 \cdot \mathrm{P}^{2}, \\
& \mathrm{Q}_{\mathrm{t}}=3.0155+0.352 \cdot \mathrm{P}-0.0037 \cdot \mathrm{P}^{2} .
\end{aligned}
$$

Figure 2 shows the dependence of the flow rate of a water-packed vacuum pump with an upstream pulsating ejector $Q=f(P)$ on the amount of vacuum with metal, rubber and textolite ball valves.

The graph shows that for an ejector with a pulsating motive flow, the recommended valve material is a metal ball.

As a result of joint mathematical processing of the experimental results, the dependences of productivity on the pulsation frequency for various vacuum values were obtained:

$$
\begin{aligned}
& \text { - for } \mathrm{P}=20 \mathrm{kPa}, \\
& \mathrm{Q}_{1}=13.515+0.0363 \cdot \omega-0.0003 \cdot \omega^{2}, \\
& \text { - for } \mathrm{P}=30 \mathrm{kPa} \text {, } \\
& \mathrm{Q}_{2}=13.965+0.0363 \cdot \omega-0.0003 \cdot \omega^{2}, \\
& \text { - for } \mathrm{P}=50 \mathrm{kPa} \text {, } \\
& \mathrm{Q}_{3}=14.265+0.0363 \cdot \omega-0.0003 \cdot \omega^{2}, \\
& \text { - for } \mathrm{P}=60 \mathrm{kPa} \text {, } \\
& \mathrm{Q}_{4}=13.366+0.0609 \cdot \omega-0.0004 \cdot \omega^{2} \text {. }
\end{aligned}
$$

Based on the resulting patterns, maximum productivity is ensured at the pulsation frequency 90-100 $\mathrm{min}^{-1}$. 


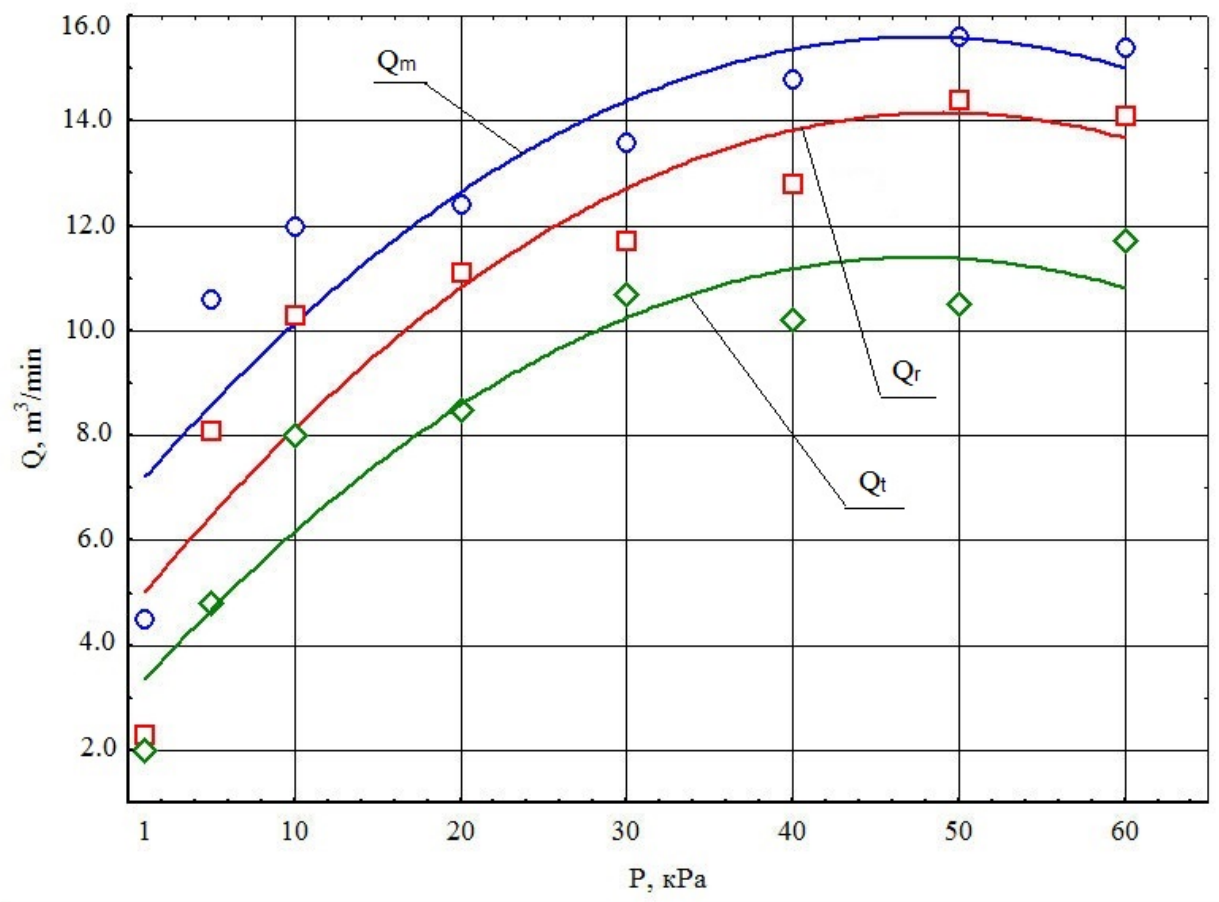

Fig. 2. Vacuum pulse ejector inlets with various ball valves: $Q_{m}$ - a metal ball; $Q_{r}$ - a rubber ball; $Q_{t}-$ textolite ball

\section{Conclusions}

The pulsating jet vacuum pumping device is widely used in all industries and agriculture for conveying various materials.

To increase the efficiency of transporting materials, an ejector with an oscillating motive flow is developed as part of a vacuum pumping device.

To describe the technological process of transporting material using an ejector with an oscillating motive flow, a theoretical relationship is presented.

The most effective performance indicators of ejectors, including the degree of pressure increase, ejection coefficient and geometric parameters, were determined during experiments.

A direct relationship was established between changes in the performance of a pulse-vacuum pumping device and valve material and magnitude of its oscillations.

An ejector with a pulsating motive flow is recommended to have a metal ball-seated valve with a pulsation frequency of 90-100 $\mathrm{min}^{-1}$.

Once applied, the proposed pulse ejector will eventually increase the transportation productivity by $14.5 \%$.

\section{References}

1. A.I. Fokin, Yu.A. Tsoi, B.G. Ziganshin, I.N. Gayaziev, Combined installation for cooling milk using artificial and natural cold, Rural mach. and equipm., 10, 11-12 (2015)

2. I.M. Gomaa, I.I. Kashapov, R.R. Khaidarov, R.F. Sabirov, F.F. Khasanova, Technological factors influence on the work efficiency of the feed grinder, BIO Web of Conf., 17, 00233 (2020)

3. N.Kh. Zinnatullin, B.G. Ziganshin, I.M. Nafikov, M.A. Lushnov, G.N. Zinnatullina, Injection mixer for solid bulk materials, Bull. of the Kazan State Agrar. Univ., 12(3), 68-71 (2017)

4. A.V. Dmitriev, B. Ziganshin, D. Khaliullin, A. Aleshkin, Study of efficiency of peeling machine with variable deck, Engin. for rural develop., 10531058 (2020)

5. A.V. Belinsky, B. Ziganshin, A. Valiev, D. Haliullin, I. Galiev, N. Adigamov, Theoretical investigation of increasing efficiency of combine harvester operation on slopes, Engin. for Rural Develop., 206-213 (2019)

6. R.I. Ibyatov, A. Dmitriev, B. Ziganshin, D. Khaliullin, A. Zinnatullina, Mathematical modeling of the grain trajectory in the workspace of the sheller with rotating decks, BIO Web of Conf., 17, 00093 (2020)

7. B.L. Ivanov, B.G. Ziganshin, R.F. Sharafeev, I.R. Sagbiev, The theory of liquid spraying by nozzles, Bull. of the Kazan State Agrar. Univ., 14(2(53)), 95-99 (2019)

8. R.R. Lukmanov, B.G. Ziganshin, Yu.Kh. Shogenov, R.R. Lukmanov et al., Engineering solution for increasing the efficiency of machine milking of cows, Bull. of the Kazan State Agrar. Univ., 11(1(39)), 77-81 (2016)

9. M.A. Lushnov, Constructing a classification scheme and evaluating devices for heat treatment of semiliquid feed mixtures, Agricult. Sci. Euro-North-East, 5(36), 65-68 (2013) 
10. A.I. Rudakov, I.R. Nafikov, B.L. Ivanov, Increasing energy efficiency of freeze drying of agricultural materials, Bull. of the Kazan State Agrar. Univ., 2(2(6)), 101-105 (2007)

11. A.A. Mustafin, B.G. Ziganshin, Analysis of the results of experimental studies of a two-rotor vacuum pump, Bull. of the Kazan State Agrar. Univ., 1(27), 63-68 (2013)

12. A.A. Mustafin, B.G. Ziganshin, R.R. Gainutdinov, I.N. Gayaziev, Method of calculating a two-rotor vacuum pump with involute gearing, Bull. of the Kazan State Agrar. Univ., 1(23), 102-104 (2012)

13. B.G. Ziganshin, I.N. Gayaziev, I.I. Kashapov, R.R. Gainutdinov, T.R. Nuriakhmetov, To the determination of the design and technological parameters of a two-rotor vacuum pump, Bull. of the Kazan State Agrar. Univ., 7(4(26)), 75-78 (2012)

14. A.I. Rudakov, N.M. Asadullin, Pulsating transportation of pseudoplastic fluids through pipes in animal husbandry, Bull. of the Kazan State Agrar. Univ., 4, 62-67 (2006)

15. A.I. Rudakov, N.M. Asadullin, Pulsating ejector (Russia), Patent 2097606 RF, MKI 6 F 04 F 5/04. No. 94009704/06. Appl. 03/15/94; publ. 27.11.97, bull. No. 33. 4 p.

16. G.N. Abramovich, T.A. Girshovich et al., Theory of turbulent jets, Ed. by G.N. Abramovich (Nauka, Moscow, 1984), 716 p. 